\title{
Review of the Effectiveness of Cellulose- and Polysulfone-Based Vitamin E-Bonded Dialysis Membranes
}

\author{
Masaharu Aritomi ${ }^{1}$ and Francesco Galli ${ }^{2}$ \\ ${ }^{1}$ Asahi Kasei Kuraray Medical Co., Ltd. Tokyo \\ ${ }^{2}$ Dept of Internal Medicine, University of Perugia \\ 1 Japan \\ Italy
}

\section{Introduction}

Reduction of the stressful effects of extracorporeal circulation is a major target in hemodialysis (HD) therapy. These effects can be attributed to the HD-associated uremic comorbidity and the oxidative and non-oxidative events that occur because of the extracorporeal treatment. Anemia, cardiovascular disease, chronic inflammation, immunosuppression and intradialytic hypotension are among the most common complications in patients receiving HD, which show a causal relationship with oxidative stress (Galli, 2002 and Del Vecchio et al., 2011). During HD, the patient's blood is repeatedly exposed to components of the extracorporeal circulation, a key component of which is the hollow-fiber dialyzer membrane. This phenomenon may lead to leukocyte and platelet activation, thereby causing oxidative stress. Moreover, bioactive contaminants, e.g., bacterial endotoxins in the dialysis fluids, to which the patient's blood is eventually exposed through the dialyzer membrane, may further sustain leukocyte activation. These variables, which are usually included under the definition of "bio-incompatibility," should be carefully monitored by dialysis centers because these variables are responsible for causing proinflammatory events, oxidative stress, and pro-thrombotic effects. Leukocyte activation and oxidative stress are also reported to cause erythrocyte damage that is further aggravated by shear stress and other mechanical injuries. Plasma proteins and lipids show signs of oxidative damage (Piroddi et al., 2007 and Galli, 2007), and this may influence the burden of uremic toxicity by inducing cell and tissue reactions, abnormal metabolism of these oxidation products, vascular and immune reactions, etc. To reduce the risk of oxidative stress and other adverse reactions that can be caused by poor biocompatibility of HD treatment, highly purified dialysis fluids and dialyzer membranes with greater biocompatibility have been developed, and are currently under investigation for further improvement.

The bio-incompatibility of prototypical dialyzer membranes made of regenerated cellulose has been associated with the contact of blood components with the hydroxyl groups of beta-D-glucose. These groups trigger intradialytic activation of the complement 
system and cause leucopenia (Rousseau et al., 1999). Biocompatibility of regenerated cellulose dialyzer membranes was tentatively improved by the chemical modification of the hydroxyl groups, e.g., by acetylation to obtain tri-acetate cellulose, or by adding polyethylene glycol (PEG) chains to obtain PEG-grafted cellulose. Another and more successful approach was the de novo development of synthetic polymer membranes such as polysulfone or polyether sulfone membranes with no bio-incompatible groups in their chemical structures (Bowry, 2002). At present, high-flux synthetic membranes are more frequently used, and represent a cost-effective solution with proven clinical superiority over cellulosic membranes (Krane et al., 2007).

Antioxidants such as vitamin C, vitamin E, and glutathione have been used as oral supplements to alleviate HD-induced oxidative stress (Galli and Azzi, 2010). The clinical course of chronic kidney disease (CKD) includes a progressive decrease in the levels of blood antioxidants such as vitamins C, vitamin E, and glutathione. As a result, CKD patients who undergo regular HD treatment show severe deficiencies, particularly of water-soluble antioxidants (such as vitamin C). Few studies, however, provided clear evidence of the clinical advantage of oral antioxidants. The most convincing evidence was obtained from the randomized clinical trial on vitamin E supplementation in HD patients. This trial, known as SPACE study (Boaz et al., 2000), showed a significant reduction (54\%) of the primary endpoint variable (a composite variable including myocardial infarction, ischemic stroke, peripheral vascular disease, and unstable angina) and $70 \%$ reduction in myocardial infarction.

One of the most original approaches for antioxidant therapy in HD involved the use of vitamin $\mathrm{E}$ as a lipophilic modifier of the surface of the dialysis membrane. The bonded vitamin $\mathrm{E}$ on the surface of the dialyzer membrane is expected to reduce the production of reactive oxygen species (ROS) at the site where blood cells are exposed to the dialyzer membrane, thereby providing the blood cells with antioxidant protection in a timely and targeted manner. Two generations of vitamin E-bonded dialyzers based on this concept, namely, cellulosic- and polysulfone-based membrane dialyzers, have been successfully developed and launched in the market over the last 2 decades. This chapter provides a review of the literature and a critical examination of the available clinical data on vitamin Ebonded dialyzer membranes.

\section{Cellulose-based vitamin E-bonded dialyzer membranes}

\subsection{Development}

In 1990, cellulose-based vitamin E-bonded dialyzer membranes (Excebrane) were developed and introduced into the market by Terumo Corporation. The base membrane was made of regenerated cellulose hollow fiber, and the surface of the hollow fiber was covalently modified by hydrophilic polymers as well as by oleic alcohol. Vitamin E ( $\alpha$-tocopherol) was then bonded to oleic alcohol via hydrophobic interaction. Sasaki et al., 2000, reported the outline of the production process as well as the results of in vitro and in vivo studies on Excebrane, which showed much better biocompatibility than the original regenerated cellulose membrane.

\subsection{Clinical outcomes}

Early trials using Excebrane indicated that its use would have positive clinical effects (Galli, 2002). The potential therapeutic effects of using this membrane in HD were reduction of oxidative stress and inflammation. Cardiovascular endpoints were investigated in several small clinical trials and in a meta-analysis of 14 peer-reviewed articles by Sosa et al., 2006; these studies concluded that Excebrane treatment was associated with a significant decrease in 
the plasma levels of lipid-peroxidation biomarkers, thereby suggesting the potential benefit of these membranes in clinical usage. Actually, these biomarkers have a cause-effect relationship with low density lipoprotein (LDL) damage and endothelial dysfunction in CKD. Preliminary evidence of better management of uremic anemia by these dialyzer membranes was obtained in the pioneering studies by Usberti et al., 2002; Nakatani et al., 2003; and Kobayashi et al., 2003. These authors found improved erythrocyte life span and rheology in patients receiving HD with Excebrane. A larger multicenter study (172 patients) reported by Cruz et al., 2008, confirmed that the impact of Excebrane dialyzers on anemia parameters was better than that of other high-flux biocompatible dialyzer membranes, including cellulose acetate, polysulfone, and polymethylmethacrylate.

Other reports demonstrated positive effects such as (1) decreased oxidative stress (Tarng et al., 2000; Miyazaki et al., 2000; Clermont et al., 2001; Westhuyzen et al., 2003; Bufano et al., 2004; Mydlik et al., 2004; Calò et al., 2004; Yang et al., 2006; and Odetti et al., 2006), (2) suppression of leukocyte activation (Omata et al., 2000; Zaluska et al., 2001;Pertosa et al., 2002; Tsuruoka et al., 2002; Libetta et al., 2004; and Kojima et al., 2005), (3) dosage reduction of anticoagulants (Huraib et al., 2000), (4) improved biocompatibility (Yoshida et al., 2002), and (5) decreased levels of advanced glycation end products (AGEs) (Baragetti et al., 2006). Moreover, a recent study reported by Kirmizis D et al., 2010, showed that the levels of inflammatory markers such as C-reactive protein (CRP), interleukin (IL)-6, and soluble intercellular adhesion molecule (sICAM)-1 in 35 patients treated with Excebrane were lower than those in the baseline evaluation carried out with conventional low- or middle-flux dialyzers. Whereas, no change was observed throughout the experimental period in a matched control group of 25 patients who underwent treatment with the unmodified dialyzers.

\section{Polysulfone-based vitamin E-bonded dialyzer membranes}

\subsection{Development}

Synthetic membrane dialyzers have been developed by many manufacturers in the past decades to achieve higher depurative and biocompatibility standards. Polysulfone has shown better performance among these synthetic biomaterials; as a result, it has become popular in clinical practice (Bowry, 2002). In order to achieve the synergistic effect of the biocompatibility of synthetic membranes and the antioxidant activity of vitamin E, polysulfone-based vitamin E-coated membranes were developed and introduced in the market in 1998. These dialyzers were initially developed by Terumo Corporation; subsequently, they were produced using a new technique and are now manufactured by Asahi Kasei Kuraray Medical Co. Ltd., with the membrane name of VitabranE. Preliminary in vitro analyses on these membranes were reported by Sasaki, 2006; the antioxidant capacity of VitabranE was recently confirmed and quantified by Floridi A, et al., 2009, by means of in vitro re-circulation tests carried out on mini-module dialyzers. These tests, which used an unbiased procedure, showed that at least one-third of the vitamin E present on the membrane participates in the one-electron transfer reaction with transition metals. This reaction, together with scavenging of peroxyl radicals, characterizes the antioxidant mechanism of vitamin $\mathrm{E}$ and is of putative relevance in its biological function.

\subsection{Improvement of anemia}

On the basis of the early evidence obtained in the Excebrane studies described in section 2.2, VitabranE was proposed as a more biocompatible dialyzer membrane to achieve a better control of anemia in HD patients. This aspect was investigated in clinical trials that 
compared VitabranE with polysulfone membranes. A pilot study by Andrulli et al., 2010, carried out with a 2-arm randomized controlled design showed that 8-month treatment with VitabranE decreased the erythropoiesis-stimulating agent (ESA)-resistance index (ERI); ERI is calculated as the ratio between ESA dosage $(\mathrm{IU} / \mathrm{kg} / \mathrm{w})$ and hemoglobin levels $(\mathrm{g} / \mathrm{dL})$. In the primary analysis, ERI showed no significant deference between the groups treated with VitabranE and the control polysulfone membrane. However, in the secondary analysis where the baseline parathyroid hormone (PTH) and serum vitamin E levels were included as covariates, the ERI was significantly decreased in the group treated with VitabranE than in the group treated with the control polysulfone membrane. Therefore, the authors concluded that VitabranE membranes have potentially beneficial effects on ERI of HD patients. This conclusion was confirmed in another and more recent multicenter study by Panichi et al., 2011. In this report, a crossover design was employed and 62 HD patients from 13 dialysis units were randomized to receive treatments with VitabranE or polysulfone dialyzer controls. The patients were studied for 2 steps of 6 months in each treatment. In this study, hemoglobin levels significantly increased after 6 months of VitabranE treatment, whereas these remained unchanged in the control group. Moreover, at the same ESA dose, the ERI was significantly lower during the VitabranE treatment period.

Further support for the findings of these randomized trials came from a pilot cross-over study by Mandolfo et al. (in press), which was conducted on patients using central venous catheters for blood access. Sixteen patients were enrolled and divided into 2 treatment groups (VitabranE dialyzer versus synthetic membrane dialyzers) and were followed for 2 periods of 6 months each. The results showed that the ERI decreased significantly in the group that received treatment with VitabranE, whereas it did not change in the control group. Hemoglobin levels were not affected; thus, the authors concluded that VitabranE membranes might help reducing the ESA dose required in anti-anemic therapy.

\subsection{Improvement of inflammatory markers}

In the report by Panichi et al., 2011, the authors observed that beside of ERI reduction, CRP and IL-6 levels decreased during VitabranE treatment, whereas no significant changes were found during the control polysulfone treatment. The study by Mandolfo et al. (in press) on catheterized patients with a high inflammatory burden also reported a better control of CRP levels during the VitabranE treatment. In these studies, a lower inflammatory response was associated with improved ERI, which is consistent with the role of chronic inflammation and oxidative stress as pathogenic factors of uremic anemia (Del Vecchio et al., 2011).

Calò et al., 2011, conducted a 1-year study on 25 HD patients to evaluate the effects of VitabranE on the biochemical markers of inflammation. The authors employed a molecular approach by using immunoblot (western blot) analysis to assess the expression of p22phox, plasminogen activator inhibitor (PAI)-1, phosphorylated extracellular signal-regulated kinase (pERK) $1 / 2$ and heme oxygenase (HO)-1 in circulating mononuclear leukocytes at the beginning of the study and after 6 and 12 months of treatment with VitabranE. The treatment with VitabranE significantly decreased the expression of inflammatory markers of p22phox, PAI-1, and pERK1/2, while the treatment increased the expression of antiinflammatory marker of HO-1.

\subsection{Reduction in the levels of oxidative stress makers}

Preliminary investigations of blood levels of advanced protein oxidation products (AOPP) and fluorescent AGEs were performed in the randomized controlled pilot trial reported by 
Andrulli et al., 2010. In this study, these protein-damaged markers were investigated as oxidative and carbonyl stress indices associated with the correction of ERI. Although IL-6 and CRP levels were not affected, the 2 indices showed better control in the patients receiving with VitabranE than in those receiving treatment with polysulfone dialyzer (Galli et al., manuscript in preparation).

Vitamin E-bonded dialyzer membranes were originally developed with the aim of reducing oxidative stress. As shown in the section 2.2, lowered lipid peroxidation was observed in clinical trials with Excebrane, a cellulose-based vitamin E-bonded membrane. In the case of VitabranE, 2 studies were conducted, considering LDL oxidation as an end point. Morimoto et al., 2005, reported that the 15 patients who received 6 months of treatment with VitabranE showed significant reduction in the levels of asymmetric dimethylarginine (ADMA), malondialdehyde low density lipoprotein (MDA-LDL), and oxidized low density lipoprotein (Ox-LDL). The levels returned to baseline when the membrane was changed to a polysulfone dialyzer membrane. In a matched control group, patients treated with polysulfone dialyzers showed no change in ADMA, Ox-LDL, and MDA-LDL levels during the entire treatment period of 18 months. The other report from Calò et al., 2011, showed that VitabranE improved the levels of inflammation markers and also reduced plasma levels of Ox-LDL, which was evaluated by enzyme-linked immunosorbent assay.

\subsection{Improvement of intradialytic hypotension}

Intradialytic hypotension (IDH) is a common clinical trait in HD patients. Matsumura et al., 2010, conducted a pilot study to assess the effectiveness of VitabranE in improving IDH. Eight IDH patients who had been receiving HD with conventional dialyzers were switched to VitabranE dialyzers, and intradialytic blood pressure (BP) was monitored regularly for 10 months. The results showed that hypotension, monitored during the session by measuring systolic $\mathrm{BP}(\mathrm{SBP})$, diastolic $\mathrm{BP}(\mathrm{DBP})$, and pulse pressure (PP), improved after changing the dialyzer membrane. Moreover, after 8 to 10 months, SBP recorded before dialysis was significantly lower than that at baseline, which suggests a stable improvement in the vascular compliance to intra- and inter-dialysis control of BP.

\subsection{Anticoagulation management}

In a recent report, Aoun et al., 2010, described the potential clinical advantages of VitabranE in anticoagulant management. In an observational trial, these authors evaluated the minimum requirement of low molecular weight heparin (LMWH) in pediatric dialysis patients. Seven children and adolescent patients received HD with VitabranE dialyzer and their LMWH dose was decreased every week without any other change in the clinical management; the findings of this study consistently indicated a lower requirement of anticoagulants, which may help reducing bleeding problems and simplifying post-dialysis hemostasis.

\section{Conclusion}

The available literature suggests that vitamin E bonding on the cellulosic membrane Excebrane can reduce oxidative stress, as assessed by the levels of lipid peroxidation markers, and improve laboratory indices of inflammation and uremic anemia. The newly developed synthetic polysulfone-based dialysis membrane VitabranE appears to produce even more relevant clinical advantages as it is a homologue of most widely used synthetic membrane dialyzers. Randomized studies carried out in the past few years, which have 
reported positive effects on ERI and anemia management, provide convincing and clinically relevant evidence. Early findings also suggest a better control of inflammatory and oxidative stress parameters. Anticoagulation and IDH are other potentially relevant aspects in the clinical application of VitabranE. These results suggest that VitabranE has superior performance than other synthetic membranes. Further and more comprehensive trials are needed for detailed verification of these clinical outcomes.

\section{References}

Andrulli, S.; Di Filippo, S.; Manzoni, C.; Stefanelli, L.; Floridi, A.; Galli, F. \& Locatelli, F. (2010). Effect of Synthetic Vitamin E-Bonded Membrane on Responsiveness to Erythropoiesis-Stimulating Agents in Hemodialysis Patients: A Pilot Study. Nephron Clin Pract. 115(1):c82-c89.

Aoun, B.; Janssen-Lozinska, Y. \& Ulinski, T. (2010). Effect of vitamin E coated dialyzers on anticoagulation requirement in hemodialyzed children. Saudi J Kidney Dis Transpl. 21(3):466-70.

Baragetti, I.; Furiani, S.; Vettoretti, S.; Raselli, S.; Maggi, FM.; Galli, F.; Catapano, AL. \& Buccianti, G. (2006). Role of vitamin E-coated membrane in reducing advanced glycation end products in hemodialysis patients: a pilot study. Blood Purif. 24(4):369-76.

Boaz, M.; Smetana, S.; Weinstein, T.; Matas, Z.; Gafter, U.; Iaina, A.; Knecht, A.; Weissgarten, Y.; Brunner, D.; Fainaru, M. \& Green, MS. (2000). Secondary prevention with antioxidants of cardiovascular disease in endstage renal disease (SPACE): randomised placebo-controlled trial. Lancet. 356(9237):1213-8.

Bowry, SK. (2002). Dialysis membranes today. Int J Artif Organs. 25(5):447-60.

Bufano, G.; Usberti, M.; Mandolfo, S.; Malberti, F.; Piroddi, M. \& Galli, F. (2004). Von Willebrand factor and autoantibodies against oxidized LDL in hemodialysis patients treated with vitamin E-modified dialyzers. Int J Artif Organs. 27(3):214-21.

Calò, LA.; Naso, A.; Pagnin, E.; Davis, PA.; Castoro, M.; Corradin, R.; Riegler, P.; Cascone, C.; Huber, W. \& Piccoli, A. (2004). Vitamin E-coated dialyzers reduce oxidative stress related proteins and markers in hemodialysis--a molecular biological approach. Clin Nephrol. 62(5):355-61.

Calò, LA; Naso, A; D'Angelo, A.; Pagnin, E.; Zanardo, M. \& Puato, M. (2011). Rebeschini M, Landini S, Feriani M, Perego A, Malagoli A, Zagatti R, Calzavara P, Cascone C, Davis PA. Molecular biology-based assessment of vitamin E-coated dialyzer effects on oxidative stress, inflammation, and vascular remodeling. Artif Organs. 35(2):E33-9.

Clermont, G.; Lecour, S.; Cabanne, JF.; Motte, G.; Guilland, JC.; Chevet, D. \& Rochette, L. (2001). Vitamin E-coated dialyzer reduces oxidative stress in hemodialysis patients. Free Radic Biol Med. 31(2):233-41.

Cruz, DN.; De Cal, M.; Garzotto, F.; Brendolan, A.; Nalesso, D.; Corradi, V. \& Ronco, C. (2008). Effect of vitamin E-coated dialysis membranes on anemia in patients with chronic kidney disease: an Italian multicenter study. Int J Artif Organs. 31(6):545-52.

Del Vecchio, L.; Locatelli, F. \& Carini, M. (2011). What we know about oxidative stress in patients with chronic kidney disease on dialysis-clinical effects, potential treatment, and prevention. Semin Dial. 24(1):56-64.

Floridi, A.; Piroddi, M.; Pilolli, F.; Matsumoto, Y.; Aritomi, M. \& Galli F. (2009). Analysis method and characterization of the antioxidant capacity of vitamin E-interactive polysulfone hemodialyzers. Acta Biomater. 5(8):2974-82.

Galli, F. (2002). Vitamin E-modified dialyzers. Contrib Nephrol. 137:95-105. 
Galli, F.; Floridi, A. \& Buoncristiani U. (2002). Oxidant stress in hemodialysis patients. Contrib Nephrol. 137:371-8.

Galli, F. (2007). Protein damage and inflammation in uraemia and dialysis patients. Nephrol Dial Transplant. 22 Suppl 5:v20-36.

Galli, F. \& Azzi, A. (2010). Present trends in vitamin E research. Biofactors 36(1):33-42.

Huraib, S.; Tanimu, D.; Shaheen, F.; Hejaili, F.; Giles, C. \& Pagayon, V. (2000). Effect of vitamin-E-modified dialysers on dialyser clotting, erythropoietin and heparin dosage: a comparative crossover study. Am J Nephrol. 20(5):364-8.

Kirmizis, D.; Papagianni, A.; Belechri, AM. \& Memmos, D. (2010). Effects of vitamin Ecoated membrane dialyser on markers of oxidative stress and inflammation in patients on chronic haemodialysis. Nephrol Dial Transplant. [Epub ahead of print].

Kobayashi, S.; Moriya, H.; Aso, K. \& Ohtake, T. (2003) Vitamin E-bonded hemodialyzer improves atherosclerosis associated with a rheological improvement of circulating red blood cells. Kidney Int. 63(5):1881-7.

Kojima, K.; Oda, K.; Homma, H.; Takahashi, K.; Kanda, Y.; Inokami, T. \& Uchida, S. (2005). Effect of vitamin E-bonded dialyzer on eosinophilia in haemodialysis patients. Nephrol Dial Transplant. 20(9):1932-5.

Krane, V.; Krieter, DH.; Olschewski, M.; Marz, W.; Mann, JF.; Ritz, E. \& Wanner, C. (2007). Dialyzer membrane characteristics and outcome of patients with type 2 diabetes on maintenance hemodialysis. Am J Kidney Dis. 49(2):267-75.

Libetta, C.; Zucchi, M.; Gori, E.; Sepe, V.; Galli, F.; Meloni, F.; Milanesi, F. \& Dal Canton, A. (2004). Vitamin E-loaded dialyzer resets PBMC-operated cytokine network in dialysis patients. Kidney Int. 65(4):1473-81.

Mandolfo, S.; Corradi, B.; Bucci, R.; Barbisoni, F.; Farina, M; Pilolli F. \& Galli, F. (in press) Evaluation of the impact of a new synthetic Vitamin E-bonded membrane on anemia and rHuEPO requirement in ESRD patients with central venous catheter: a pilot study. Int J Nephrol.

Matsumura, M.; Sasaki, H.; Sekizuka, K.; Sano, H.; Ogawa, K.; Shimizu, C.; Yoshida, H.; Kobayashi, S.; Koremoto, M.; Aritomi, M. \& Ueki, K. (2010). Improved management of intradialytic hypotension (IDH) using vitamin E-bonded polysulfone membrane dialyzer. Int J Artif Organs. 33(3):147-153.

Miyazaki, H.; Matsuoka, H.; Itabe, H.; Usui, M.; Ueda, S. \& Okuda, S. (2000). Imaizumi T. Hemodialysis impairs endothelial function via oxidative stress: effects of vitamin Ecoated dialyzer. Circulation. 101(9):1002-6.

Morimoto, H.; Nakao, K.; Fukuoka, K.; Sarai, A.; Yano, A.; Kihara, T.; Fukuda, S.; Wada, J. \& Makino, H. (2005). Long-term use of vitamin E-coated polysulfone membrane reduces oxidative stress markers in hemodialysis patients. Nephrol Dial Transplant. 20(12):2775-82.

Mydlik, M.; Derzsiova, K.; Racz, O.; Sipulova, A.; Lovasova, E.; Molcanyiova, A. \& Petrovicova, J. (2004). Vitamin E-coated dialyzer and antioxidant defense parameters: three-month study. Semin Nephrol. 24(5):525-31.

Nakatani, T.; Takemoto, Y. \& Tsuchida, AK. (2003). The effect of vitamin E-bonded dialyzer membrane on red blood cell survival in hemodialyzed patients. Artif Organs. 27(3):214-7.

Odetti, P.; Traverso, N.; Monacelli, F.; Menini, S.; Vazzana, J.; Tasso, B.; Pronzato, MA.; Robaudo, C. \& Deferrari, G. (2006). Vitamin E-coated filter decreases levels of free 4-hydroxyl-2-nonenal during haemodialysis sessions. Free Radic Res. 40(2):207-12.

Omata, M.; Higuchi, C.; Demura, R.; Sanaka, T. \& Nihei, H. (2000). Reduction of neutrophil activation by vitamin E modified dialyzer membranes. Nephron. 85(3):221-31. 
Panichi, V.; Rosati, A.; Paoletti, S.; Ferrandello, P.; Migliori, M.; Beati, S.; Bernabini, G.; Daini, R.; Casani, A.; Angelini, D.; Parrini, M.; Rossi, A.; Petrone, I.; Barsotti, G.; Donadio, C.; Donati, G.; Grazi, G.; Manca Rizza, G.; Garosi, G.; Sansoni, E.; Braccagni, B.; Sidoti, A.; Boracelli, D.; Biagioli, M.; Moriconi, L.; Finato, V.; Mannarino, A.; Grimaldi, C.; Pansa, F.; Imperiali, P.; Mura, C.; Bianchi, S. \& Bigazzi, R. (2011). A Vitamin E-Coated Polysulfone Membrane Reduces Serum Levels of Inflammatory Markers and Resistance to Erythropoietin-Stimulating Agents in Hemodialysis Patients: Results of a Randomized Cross-Over Multicenter Trial. Blood Purif. 32(1):7-14.

Pertosa, G.; Grandaliano, G.; Soccio, M.; Martino, C.; Gesualdo, L. \& Schena, FP. (2002). Vitamin E-modified filters modulate Jun N-terminal kinase activation in peripheral blood mononuclear cells. Kidney Int. 62(2):602-10.

Piroddi, M.; Depunzio, I.; Calabrese, V.; Mancuso, C.; Aisa, CM.; Binaglia, L.; Minelli, A.; Butterfield, AD. \& Galli, F. (2007). Oxidatively-modified and glycated proteins as candidate pro-inflammatory toxins in uremia and dialysis patients. Amino Acids. 32(4):573-92.

Rousseau, Y.; Carreno, MP.; Poignet, JL.; Kazatchkine, MD. \& Haeffner-Cavaillon, N. (1999). Dissociation between complement activation, integrin expression, and neutropenia during hemodialysis. Biomaterials. 20(20):1959-67.

Sasaki, M.; Hosoya, N. \& Saruhashi, M. (2000). Vitamin E modified cellulose membrane. Artif Organs. 24(10):779-89.

Sasaki, M. (2006). Development of vitamin E-modified polysulfone membrane dialyzers. J Artif Organs. 9(1):50-60.

Sosa, MA.; Balk, EM.; Lau, J.; Liangos, O.; Balakrishnan, VS.; Madias, NE.; Pereira, BJ. \& Jaber, BL. (2006). A systematic review of the effect of the Excebrane dialyser on biomarkers of lipid peroxidation. Nephrol Dial Transplant. 21(10):2825-33.

Tarng, DC.; Huang, TP.; Liu, TY.; Chen, HW.; Sung, YJ. \& Wei, YH. (2000). Effect of vitamin E-bonded membrane on the 8-hydroxy 2'-deoxyguanosine level in leukocyte DNA of hemodialysis patients. Kidney Int. 58(2):790-9.

Tsuruoka, S.; Kawaguchi, A.; Nishiki, K.; Hayasaka, T.; Fukushima, C.; Sugimoto, K.; Saito, T. \& Fujimura, A. (2002). Vitamin E-bonded hemodialyzer improves neutrophil function and oxidative stress in patients with end-stage renal failure. Am J Kidney Dis. 39(1):127-33.

Usberti, M.; Gerardi, G.; Bufano, G.; Tira, P.; Micheli, A.; Albertini, A.; Floridi, A.; Di Lorenzo, D. \& Galli, F. (2002). Effects of erythropoietin and vitamin E-modified membrane on plasma oxidative stress markers and anemia of hemodialyzed patients. Am J Kidney Dis. 40(3):590-9.

Westhuyzen, J.; Saltissi, D. \& Stanbury, V. (2003). Oxidative stress and erythrocyte integrity in end-stage renal failure patients hemodialysed using a vitamin E-modified membrane. Ann Clin Lab Sci. 33(1):3-10.

Yang, CC.; Hsu, SP.; Wu, MS.; Hsu, SM. \& Chien, CT. (2006). Effects of vitamin C infusion and vitamin E-coated membrane on hemodialysis-induced oxidative stress. Kidney Int. 69(4):706-14.

Yoshida, K.; Kitauchi, T.; Kimura, S.; Yoneda, T.; Uemura, H.; Ozono, S. \& Hirao, Y. (2002). Serum neopterin monitoring and vitamin E-modified, regenerated hemodialyzer membrane influence on biocompatibility. Artif Organs. 26(1):54-7.

Zaluska, WT.; Ksiazek, A. \& Roliski, J. (2001). Effect of vitamin E modified cellulose membrane on human lymphocyte, monocyte, and granulocyte CD11b/CD18 adhesion molecule expression during hemodialysis. ASAIO J. 47(6):619-22. 


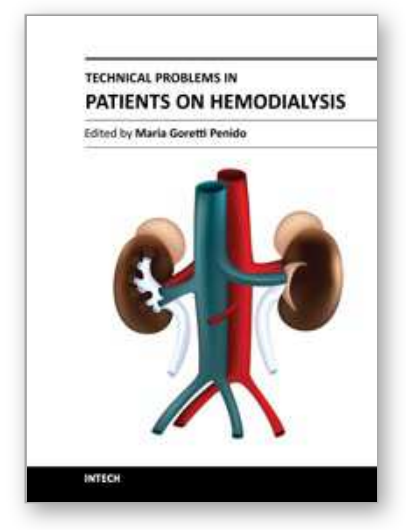

\author{
Technical Problems in Patients on Hemodialysis \\ Edited by Prof. Maria Goretti Penido
}

ISBN 978-953-307-403-0

Hard cover, 312 pages

Publisher InTech

Published online 07, December, 2011

Published in print edition December, 2011

This book provides an overview of technical aspects in treatment of hemodialysis patients. Authors have contributed their most interesting findings in dealing with hemodialysis from the aspect of the tools and techniques used.Each chapter has been thoroughly revised and updated so the readers are acquainted with the latest data and observations in the area, where several aspects are to be considered. The book is comprehensive and not limited to a partial discussion of hemodialysis. To accomplish this we are pleased to have been able to summarize state of the art knowledge in each chapter of the book.

\title{
How to reference
}

In order to correctly reference this scholarly work, feel free to copy and paste the following:

Masaharu Aritomi and Francesco Galli (2011). Review of the Effectiveness of Cellulose- and PolysulfoneBased Vitamin E-Bonded Dialysis Membranes, Technical Problems in Patients on Hemodialysis, Prof. Maria Goretti Penido (Ed.), ISBN: 978-953-307-403-0, InTech, Available from:

http://www.intechopen.com/books/technical-problems-in-patients-on-hemodialysis/review-of-the-effectivenessof-cellulose-and-polysulfone-based-vitamin-e-bonded-dialysis-membranes

\section{INTECH}

open science | open minds

\section{InTech Europe}

University Campus STeP Ri Slavka Krautzeka 83/A 51000 Rijeka, Croatia Phone: +385 (51) 770447

Fax: +385 (51) 686166 www.intechopen.com

\section{InTech China}

Unit 405, Office Block, Hotel Equatorial Shanghai No.65, Yan An Road (West), Shanghai, 200040, China 中国上海市延安西路65号上海国际贵都大饭店办公楼405单元 Phone: +86-21-62489820

Fax: +86-21-62489821 
(C) 2011 The Author(s). Licensee IntechOpen. This is an open access article distributed under the terms of the Creative Commons Attribution 3.0 License, which permits unrestricted use, distribution, and reproduction in any medium, provided the original work is properly cited. 